\title{
Extrinsic compression of coronary and pulmonary vasculature
}

\author{
Kiran Batra ${ }^{1}$, Sachin S. Saboo ${ }^{2}$, Asha Kandathil ${ }^{1}$, Arzu Canan ${ }^{1}$, Sandeep S. Hedgire ${ }^{3}$, \\ Murthy R. Chamarthy ${ }^{4}$, Sanjeeva P. Kalva ${ }^{4,5 \#}$, Suhny Abbara ${ }^{1 \#}$
}

${ }^{1}$ Department of Radiology, University of Texas Southwestern Medical Center, Dallas, TX, USA; ${ }^{2}$ Division of Cardiothoracic Imaging, Department of Radiology, University of Texas Health Science Center, TX, USA; ${ }^{3}$ Division of Cardiovascular Imaging, Department of Radiology, Massachusetts General Hospital, Harvard Medical School, Boston, MA, USA; ${ }^{4}$ Division of Interventional Radiology, Department of Radiology, University of Texas Southwestern Medical Center, Dallas, TX, USA; ${ }^{5}$ Division of Interventional Radiology, Massachusetts General Hospital, Harvard Medical, School, Boston, MA, USA

Contributions: (I) Conception and design: K Batra, SP Kalva, S Abbara; (II) Administrative support: SP Kalva, S Abbara; (III) Provision of study materials or patients: All authors; (IV) Collection and assembly of data: K Batra, SS Saboo, A Kandathil, A Canan, MR Chamarthy, SP Kalva, S Abbara; (V) Data analysis and interpretation: None; (VI) Manuscript writing: All authors; (VII) Final approval of manuscript: All authors.

\#These authors contributed equally to this work as senior authors.

Correspondence to: Kiran Batra, MD. Department of Radiology, University of Texas Southwestern Medical Center, Dallas, TX 75390-9316, USA. Email: Kiran.Batra@utsouthwestern.edu.

\begin{abstract}
Coronary artery disease from atherosclerosis induced stenosis remains the leading cause of acute coronary syndrome (ACS) and death worldwide, however extrinsic compression of coronary arteries from adjacent anatomical and pathological structures is an infrequent but important diagnosis to be aware of, especially given the nonspecific symptoms of chest pain that mimic angina in patients with pulmonary hypertension (PHT) and congenital heart disease. Non-invasive CT angiography is an invaluable diagnostic tool for detection of coronary artery compression, pulmonary artery dilatation and pulmonary vascular compression. Although established guidelines are not available for management of left main coronary artery (LMCA) compression syndrome, percutaneous coronary intervention and stent implantation remain a feasible option for the treatment, specifically for patients with a high surgical risk. Treatment of pulmonary vein or artery compression is more varied and determined by etiology. This review article is focused on detailed discussion of extrinsic compression of coronary arteries, mainly the LMCA and brief discussion on pulmonary vasculature compression by surrounding anatomical and pathological entities, with focus on pathophysiology, clinical features, complications and role of imaging in its diagnosis and management.
\end{abstract}

Keywords: Coronary; compression; pulmonary hypertension (PHT); extrinsic; aneurysm; pulmonary venous; fibrosing mediastinitis (FM)

Submitted Feb 03, 2020. Accepted for publication May 29, 2020.

doi: $10.21037 / \mathrm{cdt}-20-155$

View this article at: http://dx.doi.org/10.21037/cdt-20-155

\section{Introduction}

Extrinsic compression of coronary vessels from surrounding structures is an unusual etiology of coronary insufficiency and has been alluded to mostly in case reports and case series. Compression of coronary arteries from an enlarged pulmonary artery (PA) (1), unruptured left sinus of Valsalva aneurysm (SVA) $(2,3)$, aortic root abscess (4), prosthetic pulmonary valve placement (5), stent placement in a PA conduit (6), metastatic cardiac tumor (7), parasitic infections (8), and ventricular pseudoaneurysm (9) (amongst others) have been described as etiologies of this potentially life-threatening condition that may lead to refractory angina, persistent myocardial dysfunction and sudden cardiac death. Cases of compression of the right coronary artery (RCA) and left anterior descending 
coronary artery (LAD) have been described as a result of aortic root pseudoaneurysms and SVAs (10-12). Extrinsic compression of pulmonary vasculature is a rare but important etiology of chest pain and dyspnea that may be encountered in various clinical scenarios including chest wall deformities such as pectus excavatum and malignant infiltration and/or compressive narrowing of PA and veins. In this article, we will focus on extrinsic compression of the coronary arteries and pulmonary vessels with discussion of relevant etiopathogenesis, anatomy, clinical manifestations, potential complications, cross-sectional imaging features, and management.

\section{Coronary compression syndrome}

Coronary compression syndrome is often indistinguishable from acute coronary syndrome (ACS) - a constellation of clinical symptoms resulting from a myocardial ischemic event. Approximately $7-29 \%$ of patients with pulmonary hypertension (PHT) may report typical characteristics of angina pain, which may be attributed to coronary insufficiency from compression by a distended PA rather than atherosclerotic stenosis of the coronary artery (1).

\section{Relevant anatomy}

Typically, the left main coronary artery (LMCA) arises from the left sinus of Valsalva (LSV) and courses 2-4 mm though the aortic wall, running at about $90^{\circ}$ from the aortic ostium. It travels posterior to the pulmonary trunk, and then in the left atrioventricular groove, leftward, posterior and superior as it courses to its bifurcation or trifurcation into the left anterior descending, circumflex coronary arteries and ramus intermedius respectively (13-15). The RCA typically arises from the right sinus of Valsalva (RSV), courses anteriorly and to the right between the right atrial appendage and the $\mathrm{PA}$ and then descends vertically in the right atrioventricular sulcus. The left circumflex (LCX) is directed towards the left, travels in the atrio-ventricular groove and onto the diaphragmatic cardiac surface, usually terminating before reaching the posterior interventricular sulcus $(15,16)$.

The main PA arises from the right ventricular outflow tract and courses superior, posterior and to the left of the aorta before it bifurcates into right and left main PA inferior to the arch, at the level of the carina. The right and left pulmonary arteries further divide into 2 lobar branches each, and thereafter into segmental and sub-segmental branches.

\section{Coronary compression from enlarged $P A$}

PA dilatation is a frequent finding with an incidence of $76.6 \%$ in a cohort of severe PHT patients (17). The prevalence of greater than 50\% LMCA stenosis as a result of extrinsic compression from an enlarged $\mathrm{PA}$ is at least $6 \%$ in the overall PHT population and around $40 \%$ in the population with angina-like symptoms who had undergone a diagnostic procedure (18). Corday et al. (1957) first suggested that compression of the LMCA by a dilated PA might account for symptoms of coronary insufficiency in patients with PHT (19). Subsequently, several case reports and case series have surfaced describing LMCA compression as a complication of PHT (20-24). A 26-patient series identified a prevalence rate of $26.9 \%$ (1). An aberrant origin of the LMCA from the RSV or a rightward-positioned left coronary sinus predicts a higher risk of compression (25). Potential complications include myocardial ischemia, myocardial infarction, left ventricular dysfunction and ventricular arrhythmia (26-29). Sudden death has been reported in more than $25 \%$ of patients with PHT, possibly underscoring the importance of this diagnosis (30).

A massively enlarged main PA can occur with PHT, or in the presence of a pulmonary artery aneurysm (PAA). An enlarged PA from PHT may occur in the setting of chronic untreated left to right intracardiac shunts and chronic pulmonary thromboembolic disorder $(31,32)$. Less frequent etiologies include connective tissue disorders, portal hypertension, HIV, heritable and drug-induced or idiopathic causes (32). Small stature has also been suggested as a risk factor of coronary compression from enlarged PA (26). Incidentally, minimal or no atherosclerotic disease in the LMCA or other coronary arteries is identified in this patient group as compared to other registries, likely due to a younger age group and underlying cardiac shunts (18). The average time for diagnosis of the LMCA compression is around 5 years after the diagnosis of PHT.

A rare cause of PA enlargement is a PAA, defined as focal dilatation of a blood vessel wall encompassing all three vascular layers beyond $40 \mathrm{~mm}$ or its maximal normal caliber based on various criteria. A pseudoaneurysm on the other hand is a focal contained rupture of the artery. Both entities can be complicated by thrombosis, dissection, rupture, and/or compression of adjacent structures (33). Coronary insufficiency may occur in the absence of PHT in the setting of a low pressure PAA, and secondary to its anatomical relation with PA (34).

A rare cause of symptomatic extrinsic coronary 


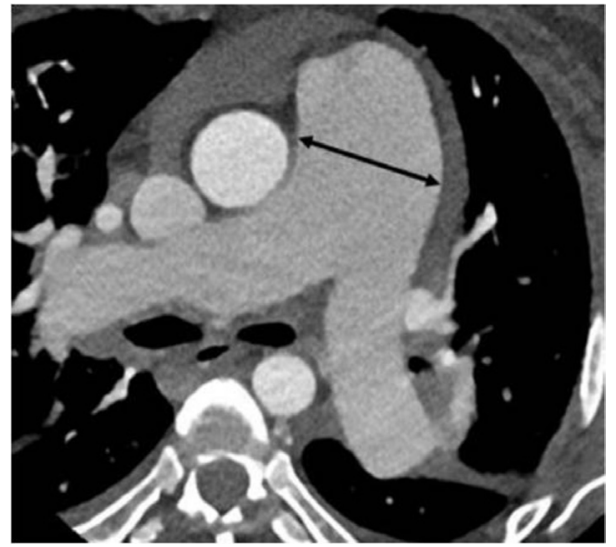

Figure 1 Measurement of main pulmonary artery diameter on CT. The diameter of the pulmonary artery is measured at the level of the bifurcation and orthogonal to the long axis. LMCA, left main coronary artery; LVOT, left ventricular outflow tract.

compression is post-surgicalrelated to surgical implantation of a sewing ring and/or pulmonary or aortic prosthetic valve strut that, based on proximity to the LMCA, can impinge on this vessel. This is usually evident in the immediate perioperative period (5).

\section{Coronary compression from unruptured SVA}

SVAs are rare cardiac anomalies that occur in $0.14 \%$ to $0.96 \%$ of the population and manifest as dilatation in the aortic wall just superior to the three cusps of the aortic valve, between the aortic valve annulus and the sinotubular ridge (35-37). Congenital aneurysms are more frequent with an incidence of $0.1-3.5 \%$ of all congenital heart defects and often caused by weakness at the junction of the aortic media and the annulus fibrosus $(38,39)$. Acquired aneurysms can occur in conditions affecting the aortic wall, such as infections (bacterial endocarditis, syphilis, or tuberculosis), trauma, or degenerative disease (atherosclerosis, connective tissue disorders, or cystic medial necrosis).

SVAs most frequently originate from the right coronary sinus (94\%), followed by the noncoronary sinus (5\%) and left coronary sinus (1\%) (40). Unruptured SVAs are typically symptom free and clinical presentation is usually due to rupture or fistulization, however a number of case reports have been published in recent years describing compression of coronary arteries, particularly the LMCA, usually involving the left coronary sinus. This typically results in angina, myocardial infarction or even sudden cardiac death
$(41,42)$, thus prompt diagnosis and management are critical.

\section{Imaging findings}

Multidetector cardiac CT is a comprehensive tool for evaluating the degree of LMCA compression, angulation of the LMCA relative to the sinus of Valsalva, and evaluation of biventricular functions. On CT, the main PA artery diameter is measured at the PA bifurcation level, orthogonal to its long axis and $3 \mathrm{~cm}$ from the root (28) (Figure 1). The sex-specific cutoff value for main PA for men is $29 \mathrm{~mm}$ and for women, $27 \mathrm{~mm}$ using the $90^{\text {th }}$ percentile. The ratio of the main PA to the ascending aorta diameter is 0.9 for both genders (43). The main PA diameter greater than $40 \mathrm{~mm}$ is identified as a strong predictor of the presence of extrinsic compression of LMCA with a sensitivity of $83 \%$ and a specificity of $70 \%$ in patients with PHT and angina $(1,18)$.

Additionally, a PA/aortic ratio of more than 1.5 and a PA diameter index of $24 \mathrm{~mm} / \mathrm{m}^{2}$ portend as risk predictors for LMCA stenosis greater than $50 \%$ with $>70 \%$ sensitivity and $68-70 \%$ specificity (18). However, not all symptomatic patients with compression of the LMCA greater than 50\% have a main PA diameter of greater than or equal to $4 \mathrm{~cm}$ and vice versa, suggesting additional anatomic variables that affect the relationship between the LMCA and the PA.

As the PA enlarges, it causes inferior displacement of LMCA and decrease in the take off-angle of the artery from the aorta. Kajita et al. evaluated angiographic determinants of LMCA extrinsic compression and found that a mean LMCA take off angle of $23^{\circ}$ (compared with an angle of $70^{\circ}$ in the control group) resulted in inferior displacement of the LMCA adjacent to a dilated PA (25). Another report found that a LMCA angle of $<30^{\circ}$ relative to the left sinus of Valsalva increased the likelihood of significant myocardial ischemia (29) (Figure 2). A strict relationship between mean PA pressure and LMCA compression has not been demonstrated.

Four anatomic categories of patients have been described, based on the relationship between the main PA and LMCA (Table 1). The normal distance between the main PA and LMCA is greater than $1 \mathrm{~mm}$. The LMCA is considered contiguous with the main $\mathrm{PA}$ if the distancePA is less than $1 \mathrm{~mm}$ but the artery is not indented or compressed. The LMCA is considered dislocated if the take-off angle of LMCA from the aorta is $\angle 60^{\circ}$ irrespective of lumen stenosis. Stenosis is considered significant if luminal diameter decreases $>50 \%$ (Figures 3,4). Selective coronary angiography is indicated when dislocation or significant 
stenosis pattern is identified on coronary computed tomography angiogram (CTA). Coronary angiography may also be considered in the presence of symptoms, even when LMCA is contiguous with main PA (Figure 5).

Chest radiograph may depict abnormal cardiomediastinal silhouette depending on the location, size, and presence or absence of rupture of SVA. SVAs vary in size from subtle dilatation of an aortic sinus to saccular outpouchings from the body or summit of the sinus. On 3D CT reconstructions, the sinus of Valsalva is measured in double short axis at the aortic root from the coronary sinus to its opposite trigone for accurate measurement (Figure 6). The end diastolic measurement of sinus of Valsalva is $3.2 \pm 0.6 \mathrm{~cm}$ for men and $2.9 \pm 0.5 \mathrm{~cm}$ for women (44).

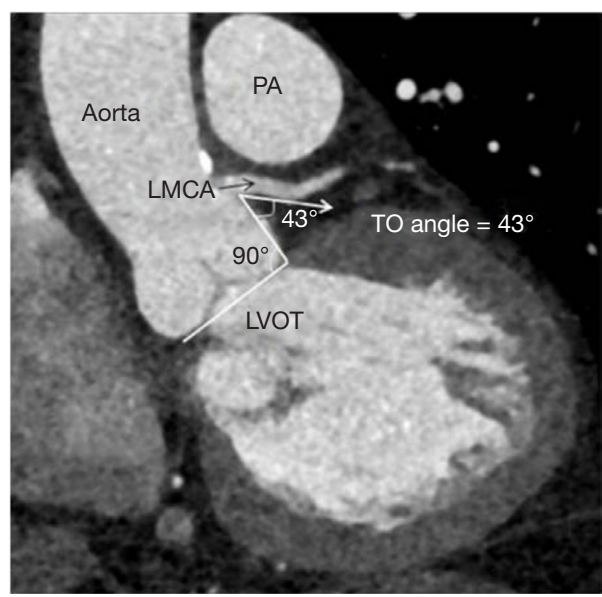

Figure 2 The oblique coronal multi planar reformat CT image adjusted for the best visualization of the proximal left main coronary artery demonstrates the measurement of its take-off (TO) angle. The angle between two planes (1-orthogonal to the aortic annulus and 2-along the proximal left main coronary artery) is measured.

\section{Management}

The appropriate management of symptomatic patients with extrinsic compression of the LMCA secondary to PHT is not well established. Invasive approaches aimed at establishing adequate blood flow to the undersupplied myocardial tissue territories include percutaneous coronary intervention (PCI) and coronary artery bypass graft (CABG). PCI is minimally invasive and may include cardiac catheterization, balloon angioplasty and stent placement for vessel recanalization. The procedure has good long-term efficacy and safety and is preferred over CABG because of risks related to general anesthesia and cardiopulmonary bypass in patients with severe pre-capillary PHT (24). Stent selection and size is based on the vessel diameter, atherosclerotic changes in the coronary artery, and risk of bleeding complications. Drug eluting stent is preferred over bare metal stent, and the latter is favored if there is a prolonged risk of bleeding with dual oral anticoagulant and antiplatelet therapy (45). Rate of restenosis is around 11\% and occurs from mechanical recoil, recompression, and instent neo-intimal hyperplasia but can be treated successfully with repeat PCI (18). CABG is a more invasive operation requiring blood vessels reimplantation from another part of the body to bypass the stenosed or occluded coronary segment. This surgery is preferred if concomitant operative intent to treat the underlying cause of PHT is being already considered (32).

In patients with a congenital defect and potentially reversible PHT, the correction of the congenital defect has been shown to decrease the progression of LMCA compression (46). Surgical treatments with symptomatic and prognostic benefits include atrial septostomy, reduction plasty of the PA, and lung transplantation.

There are currently no established guidelines for the management of PAAs. However, surgery remains the

Table 1 Four anatomic categories, based on the relationship between the main PA and LMCA

\begin{tabular}{lcccc}
\hline Findings & Normal & Contiguous & Dislocation & Significant stenosis \\
\hline Minimal distance between the MPA and the LMCA & Greater than $1 \mathrm{~mm}$ & Less than $1 \mathrm{~mm}$ & Less than $1 \mathrm{~mm}$ & Less than $1 \mathrm{~mm}$ \\
Indentation/compression of LMCA & - & - & + \\
Take-off angle of the LMCA, $<60$ degree & - & - & + \\
Stenosis of greater than $50 \%$ & - & + & + \\
\hline
\end{tabular}

MPA, main pulmonary artery; LMCA, left main coronary artery. 

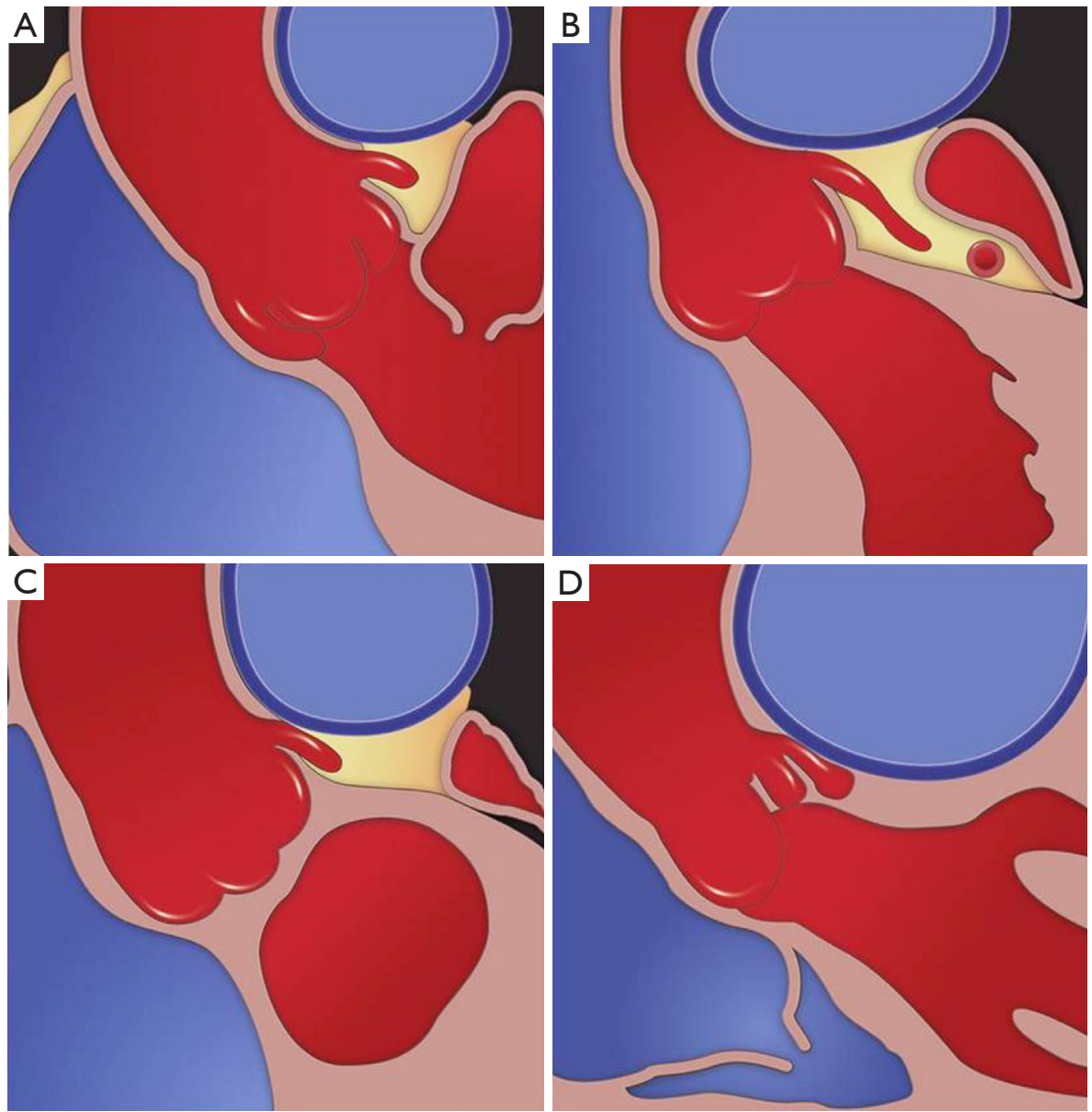

Figure 3 The illustration of the relationship between LMCA and main PA detected on cardiac CTA as categorized in four different groups. (A) Normal distance; (B) contigous; (C) dislocation; (D) significant stenosis. LMCA, left main coronary artery; CTA, computed tomography angiogram.

cornerstone of therapy for main PA lesions. Surgical intervention is considered if the aneurysm is $55 \mathrm{~mm}$ or greater, if the rate of dilatation is greater than $5 \mathrm{~mm}$ in 6 months, or if complications such as compression of adjacent structures, thrombosis, dissection, or rupture occur (47). Coronary revascularization may not be necessary if LMCA compression resolves after aneurysm surgery.

Aneurysms of the left sinus of Valsalva are extremely rare, and compression of the coronary artery resulting in coronary insufficiency is an uncommon complication (Figure 7). Surgical repair is required in cases of unruptured but symptomatic aneurysms causing ventricular outflow tract obstruction, coronary ostia obstruction, or malignant arrhythmias (42). The timing for intervention for unruptured aneurysms is still debatable as patients with unruptured, stable, or asymptomatic SVAs are usually followed clinically and with serial imaging. However, current guidelines recommend surgical correction if sinuses exceed $5.5 \mathrm{~cm}$, or $4.5 \mathrm{~cm}$ in the setting of connective tissue disease. Repair should also be considered when the growth rate exceeds $0.5 \mathrm{~cm} /$ year (48). When surgically treated, unruptured SVA repair includes aneurysm exclusion and, in some cases, replacement of the aortic valve, saphenous vein bypass grafting or a combination there of (49).

\section{Pulmonary venous compression}

In the majority of patients, there are four pulmonary veins. The right superior vein drains the right upper lobe and right middle lobe, right inferior vein drains the right lower lobe, the left superior vein drains the left upper lobe and lingula, and the left inferior vein drains the left lower 

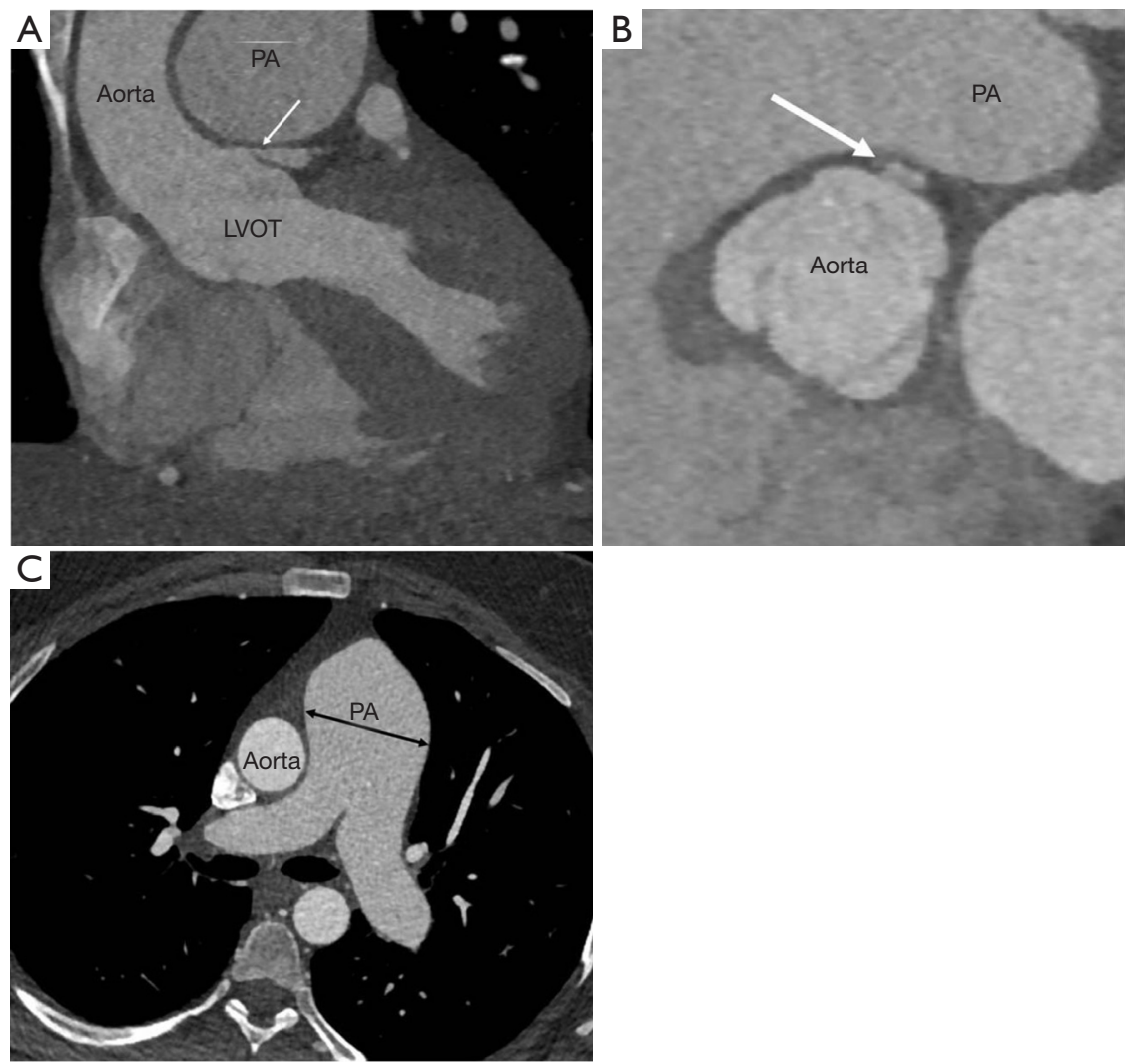

Figure 4 Coronal and axial oblique multi planar reformat image (A) and (B) show significant left main coronary artery stenosis due to compression by an enlarged main pulmonary artery $(\mathrm{C})$ in a 27 -year-old man with pulmonary hypertension. LVOT, left ventricular outflow tract.

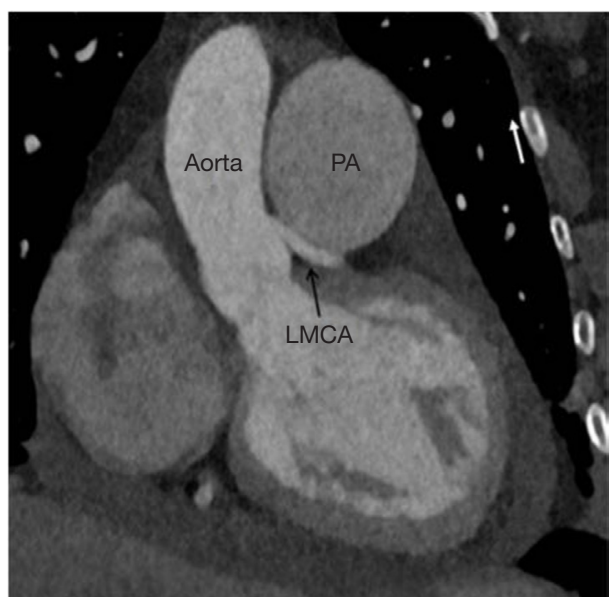

Figure 5 Oblique coronal multiplanar reformat CTA depicts dislocation of LMCA due to enlarged main PA. The main PA measures $5 \mathrm{~cm}$ and the take-off angle is less than 30 degree. No luminal narrowing of the left main coronary artery is noted. LMCA, left main coronary artery; CTA, computed tomography angiogram. lobe. Variant anatomy includes a common pulmonary vein draining the left lung and accessory veins draining the right middle or right upper lobe (50). Near the insertion site into the left atrium, the pulmonary veins are intrapericardial and are surrounded by a sleeve of pericardium and myocardium.

Pulmonary vein compression has been described in a variety of clinical scenarios. Malignant lesions include bronchogenic carcinoma, esophageal tumors, metastases and lymphoma (51). Inflammatory lesions such as fibrosing mediastinitis, sarcoidosis, tuberculosis have been described (51-54). Bronchogenic cyst, aneurysmal dilatation of the aorta and PA, and post-surgical compression following implantation of a left atrial appendage occlude, as well as chest wall deformities such as pectus excavatum (55-59).

Malignant tumors can infiltrate as well as extrinsically compress the pulmonary veins. Infiltration of the intrapericardial portion of the pulmonary veins upstages a bronchogenic carcinoma to a T4 lesion, alters surgical planning, and decreases survival (60) (Figure 8). In 

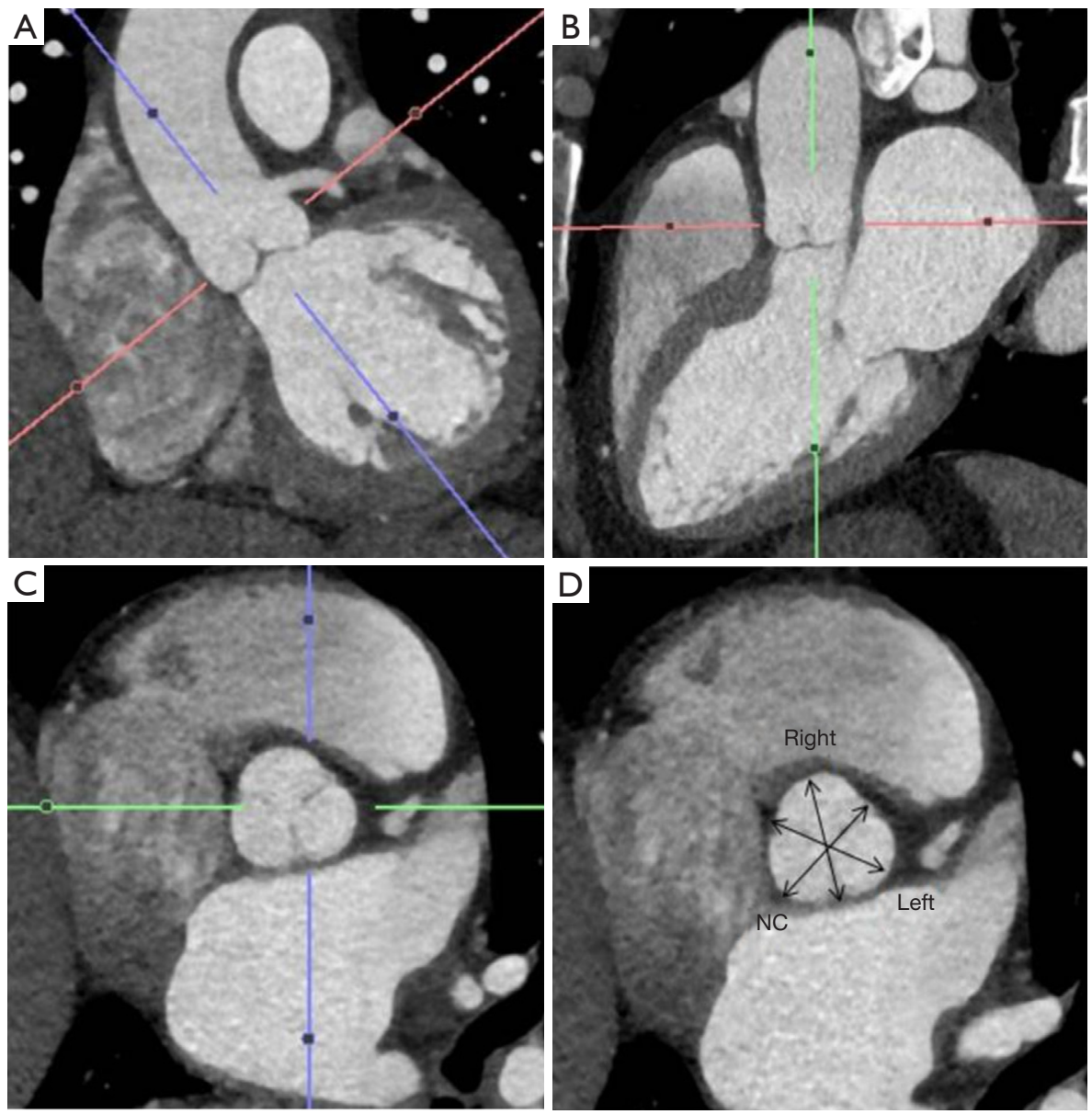

Figure 6 The measurement of sinus of Valsalva. The cross-cursors should be aligned orthogonal to aortic annulus on coronal (A) and sagittal (B) views in order to obtain double oblique short axis image of sinus of Valsalva (C), three linear distances for each sinus of Valsalva are measured (D).

patients with adequate epicardial and mediastinal fat, the pericardium may be visible and can be used to distinguish the intrapericardial from extrapericardial portion of the pulmonary vein. If the pericardium is not visible, infiltration of the epicardial fat or obliteration of the pulmonary vein ostium is suspicious for intrapericardial invasion. Choe et al. found that obliteration of the ostia by bronchogenic carcinoma is a reliable sign of intrapericardial extension for the superior pulmonary veins but a less reliable sign for inferior pulmonary veins (61). In patients with equivocal findings of pulmonary vein compression on chest CT, pulmonary vein evaluation can be performed with MRI using white-blood cine imaging, contrast-enhanced venography and phase-contrast velocity mapping (62). Indirect evidence of pulmonary vein compression includes asymmetric pulmonary edema due to venous congestion. Pulmonary edema confined to the territory drained by the occluded pulmonary vein manifests as progressive local interstitial thickening and airspace opacities (63). On CT, disruption of pulmonary venous drainage results in increased pulmonary venous pressure, leading to decreased pulmonary perfusion which can be mistaken for pulmonary thromboembolism. It can progress to pulmonary infarction which manifests as airspace opacities with internal lucencies (64).

Sternal depression in patients with pectus excavatum distorts and displaces the heart (Figure 9). Although the right ventricle is the most frequently compressed chamber, there have been reports of right and left atrial, left ventricular, and pulmonary vein compression. Pulmonary vein angle, as determined by measuring the angle between the respective pulmonary veins and patient's midline on chest CT, has been correlated with the severity of sternal depression (65). 

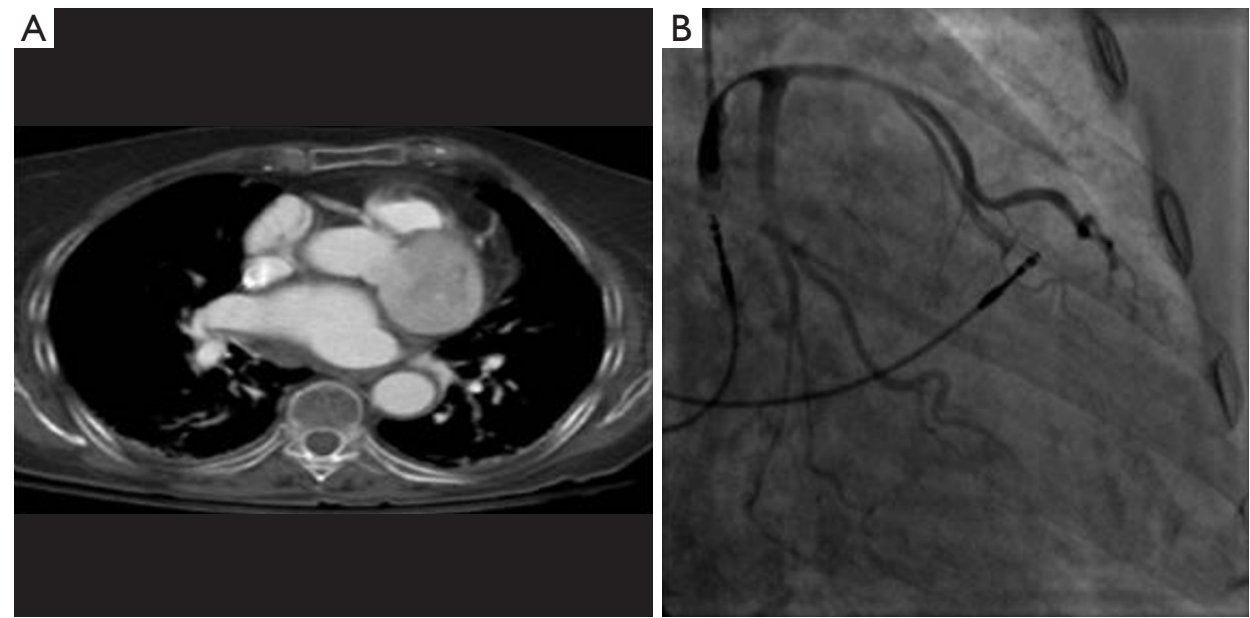

Figure 7 Reprinted with permission from reference. (A) Pre-operative imaging using computed tomographic angiography revealing giant aneurysm of the left sinus of Valsalva; (B) selective left main coronary artery angiogram revealing extrinsic compression of the left main coronary artery by the giant aneurysm of left sinus of Valsalva.
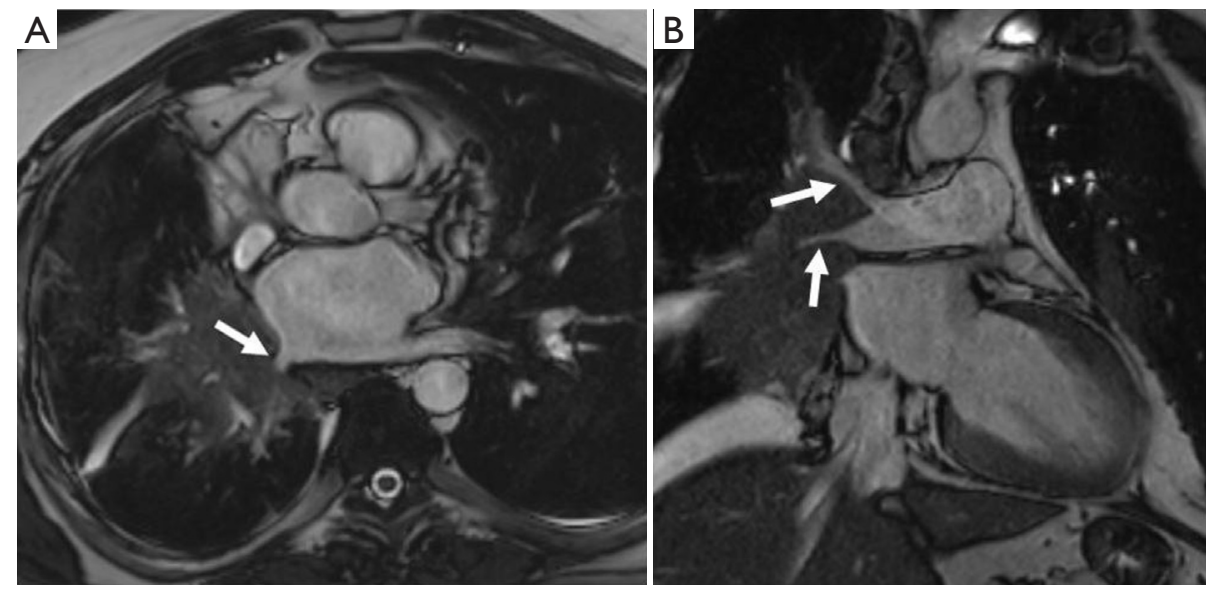

Figure 8 Axial Steady state free precession (SSFP) image in a patient with right hilar mass (small cell lung cancer) shows total occlusion of the right lower lobe pulmonary vein (arrow, A), coronal cardiac SSFP MRI image of the same patient demonstrates encasement and narrowing of the right sided lobar pulmonary arteries (arrows, B).

Treatment aims at resolving the cause of compression, often comprising antineoplastic therapy including radiation and chemotherapy, though percutaneous intervention, including endovascular stenting, has been described (63).

\section{PA compression}

Acquired PA compression is a rarely reported entity in adults. Compression of the right or left branch of the PA, without involvement of the main $\mathrm{PA}$, is more common. Extrinsic PA compression, while rare, can result from a mediastinal tumor, including lymphoma, germ cell tumors, thymoma, carcinoid tumor, lung cancer, and mediastinal metastatic disease (66-68). More rare causes of compression have been reported secondary to mediastinal sarcoma (69), mesothelioma of the pericardium (70), and sternal tumors. Non-neoplastic etiologies include infection/inflammatory causes such as FM, mediastinal cyst, thoracic aortic aneurysm, calcified pericardial ring encircling the PA above pulmonary valve level, and compression by short saphenous venous CABG (71-74). Amongst mediastinal neoplasms, teratomas and Hodgkin's disease are the most 

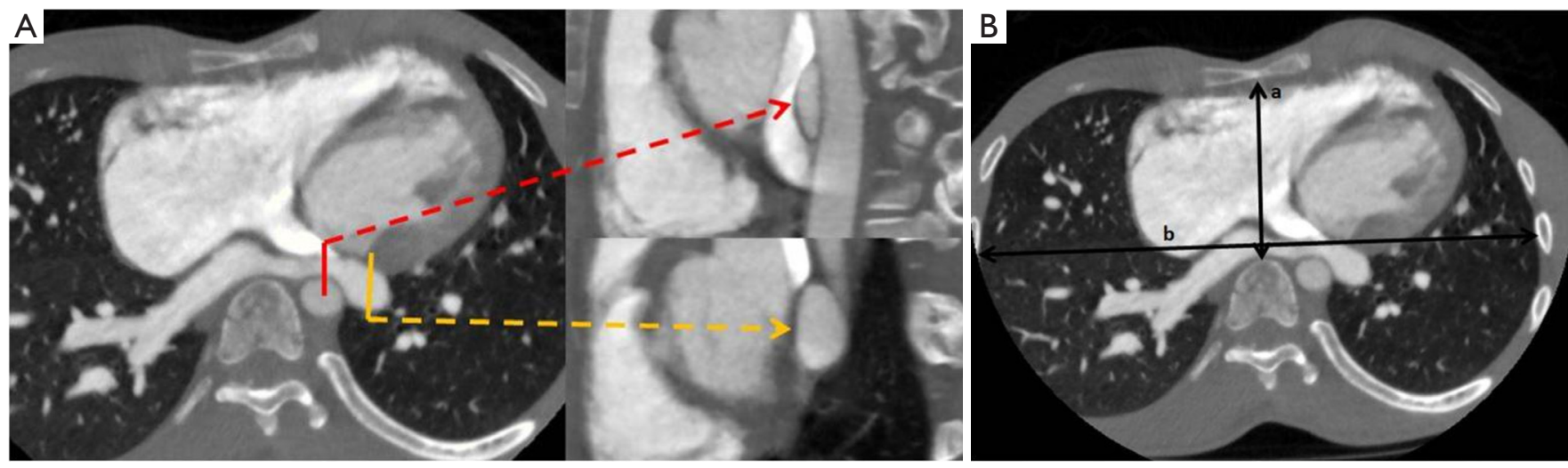

Figure 9 Axial EKG-gated cardiac CT for the evaluation of pulmonary vein stenosis in a patient with history of prior pulmonary vein ablation demonstrates the compression of the left common pulmonary vein between descending aorta and coronary sinus. The proximal portion of the pulmonary vein (A red arrow) is mildly narrowed compared to distal part (yellow arrow); (B) the vertebral column is located more anteriorly resulting in decreased anteroposterior diameter of the chest and increased Haller index $(\mathrm{a} / \mathrm{b})$.
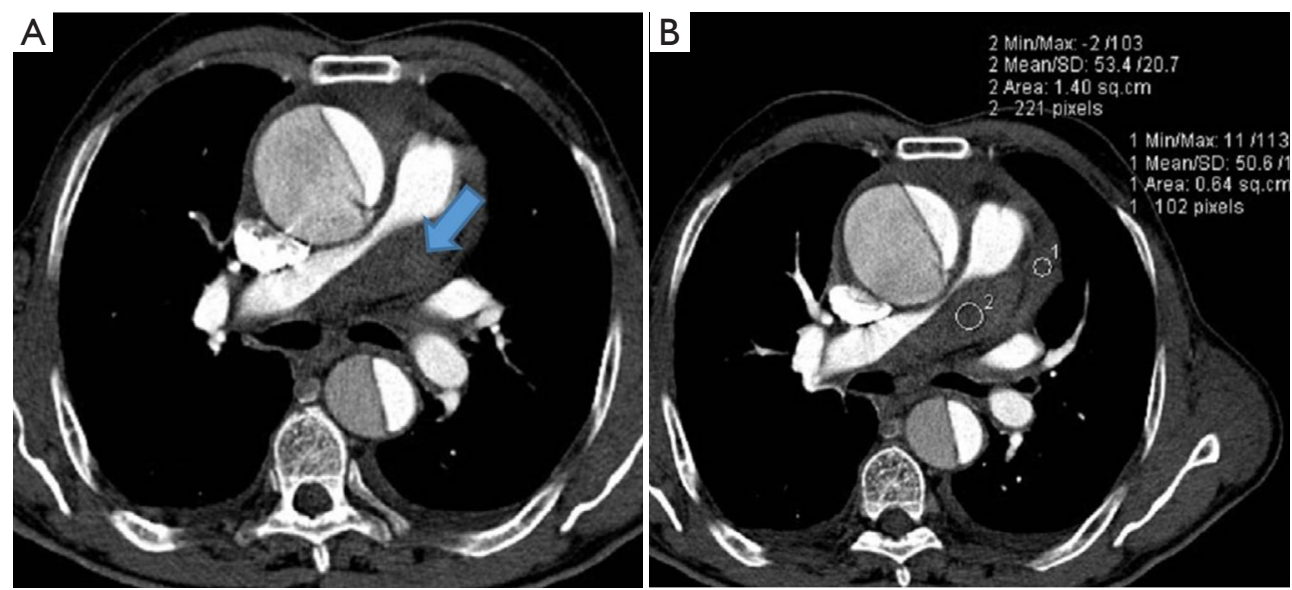

Figure 10 Axial contrast enhanced chest CT revealed (A) extrinsic compression of main and proximal right pulmonary artery by hematoma in left pulmonic recess of transverse sinus (blue arrow) with HU (Hounsefield unit) of 53, note of Type A aortic dissection and pericardial hematoma (HU 50) in (B).

common cause of extrinsic pulmonic compression. It is rare for mediastinal neoplasms to compress the PA enough to produce hemodynamically significant obstruction and is likely due to tendency of mediastinal tumors to enlarge laterally. This is more likely to result in superior vena cava obstruction (74). Extrinsic compression of PA or its central branches manifests on cross sectional imaging as compression, displacement, distortion, extrinsic indentation, luminal narrowing and in severe cases as slit-like lumen or complete occlusion (Figure 10). Chronic compression can secondarily result in right ventricular hypertrophy, as well as pulmonary and tricuspid valvular regurgitation (70).

Chest pain and dyspnea are the most common clinical manifestations of PA compression. Systolic ejection murmur is the most frequent physical examination finding (73). Clinical manifestations can overlap with acute pulmonary embolism (69).

Extrinsic compression of the main and branch PAs due to neoplastic etiology is a rare cause of right ventricular hypertension which can result in right ventricular failure. Right heart catheterization with pulmonary angiography can confirm this diagnosis and assess severity of the obstruction. CT and MRI are less invasive but nevertheless accurate methods of diagnosis (Figure 11). 2D and 3D transthoracic 


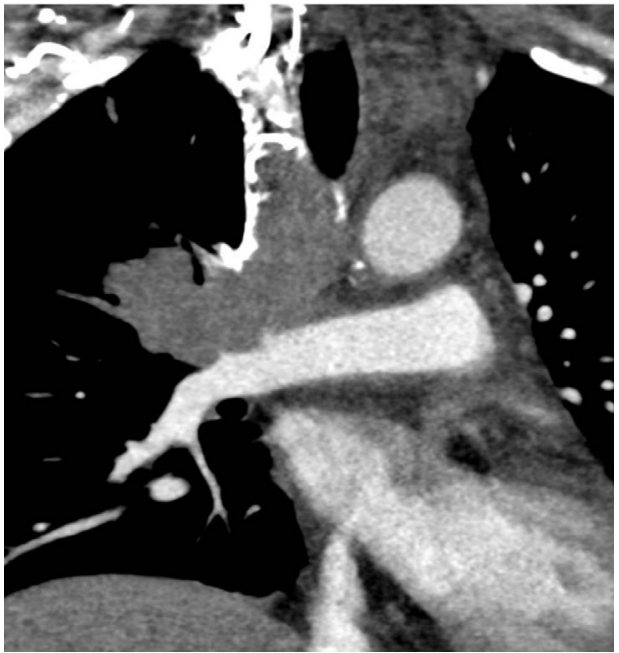

Figure 11 Post-contrast coronal chest CT reveals non-small lung cancer related large conglomerate soft tissue mass centered in the right superior mediastinal region causing complete obstruction of the right upper lobe pulmonary artery, irregular compression of the distal right pulmonary artery, near complete obstruction of the right upper lobe bronchus and invasion of SVC. echocardiogram (TTE) play pivotal roles as non-invasive tools for detection of hemodynamic compromise resulting from the pulmonary flow obstruction (68).

FM is an uncommon, progressive, benign inflammatory process related to proliferation of fibrotic tissue in the mediastinum. The localized and infiltrative subtype is more common and is frequently associated with granulomatous infection such as Histoplasma capsulatum. The diffuse form can be idiopathic or associated with autoimmune entities $(75,76)$. Two distinct radiological subtypes are seen. On CT or MRI, granulomatous FM typically manifests as localized and infiltrative heterogeneously enhancing middle mediastinal soft tissue, usually with stippled or dense calcifications. The non-granulomatous subtype presents as diffuse and infiltrative mediastinal soft tissue involving multiple compartments (Figure 12). Calcifications are typically absent in this subtype $(75,77)$.

FM characteristically presents on imaging with compression or obstruction of mediastinal vasculature and/ or the tracheobronchial tree and esophagus. FM-related obstruction of the central pulmonary arteries is typically
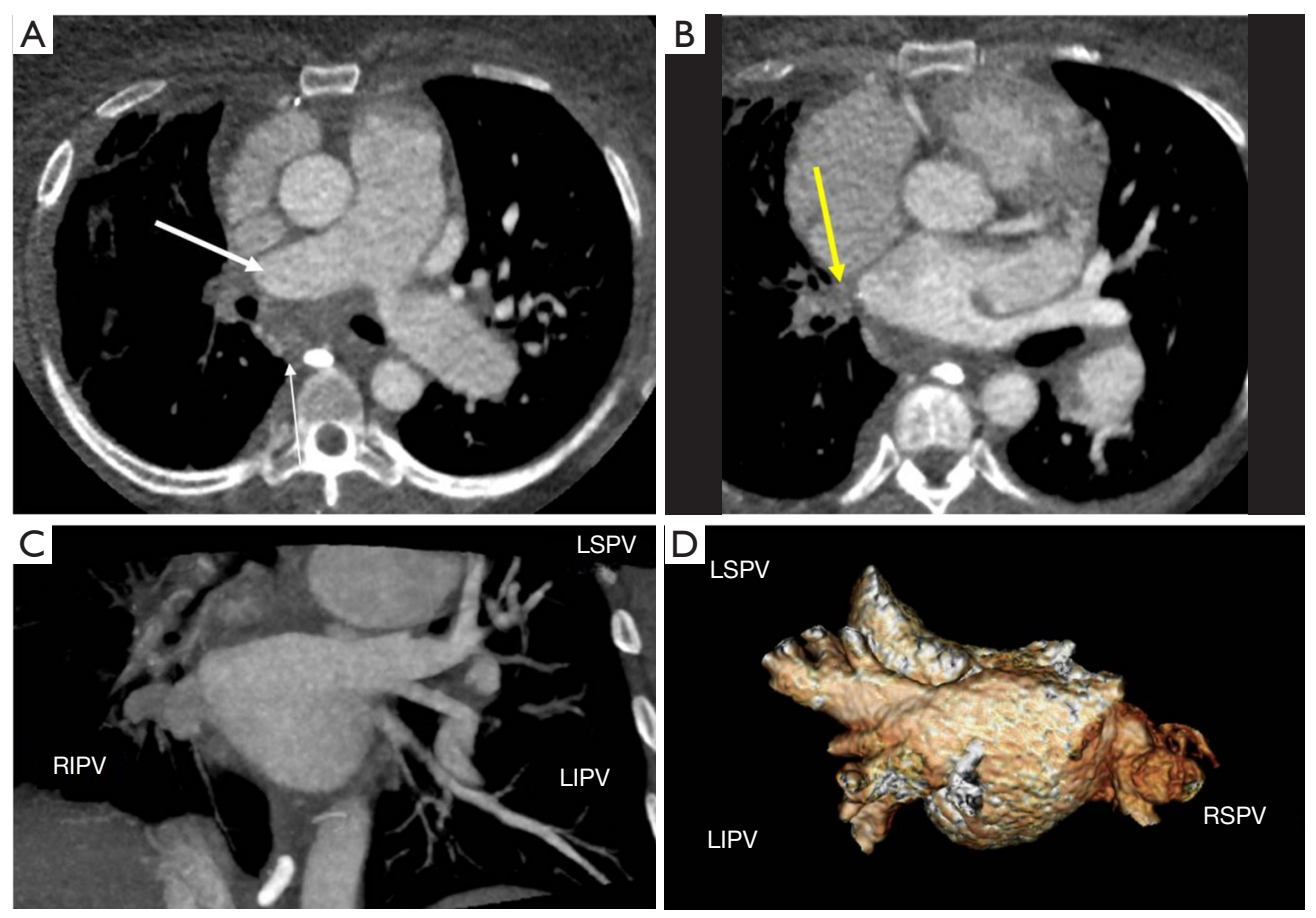

Figure 12 (A,B) Axial chest CT angiography, (C) coronal maximum intensity projection (MIP) image and (D) volume rendered 3 dimensional image show complete occlusion of right main pulmonary artery (white arrow) and right superior pulmonary vein (yellow arrow) due to right hilar and subcarinal ill defined soft tissue in a 40-year-old man with fibrosing mediastinitis. Note patent left sided pulmonary veins and tiny bronchial collaterals (thin arrow). LSPV, left superior pulmonary vein; RSPV, right superior pulmonary vein; LIPV, left inferior pulmonary vein. 
unilateral. It is characterized by hypoattenuating narrowing or obliteration of pulmonary vasculature due to surrounding soft tissue. This can result in hypoperfusion and mosaic attenuation on CT secondary to reduced vascularity of the affected lung parenchyma (77). Chronic PA compression can result in compensatory bronchial artery dilatation (78). MR angiography may demonstrate an attenuated $\mathrm{PA}$, suggesting a chronic occlusive thrombus or periarterial fibrosis (76). Chronic PA occlusion due to FM may result in pulmonary infarction. Chronic pulmonary arterial hypertension and right ventricular failure can occur as a terminal complication of FM. Note that FM should be distinguished from chronic PA narrowing related to chronic thromboembolism, as these conditions can have a similar clinical presentation, but distinctly different management (79).

\section{Clinical management}

The clinical management of extrinsic PA compression is directed towards treatment of the underlying disease. Management of PA compression secondary to an advanced malignancy aims to provide symptomatic relief. Intervention such as stenting or angioplasty is considered in cases of right ventricular dysfunction, severe pulmonary valvular regurgitation, or worsening hemodynamics (80). A neoplastic etiology may benefit from surgery, radiation, or chemotherapy, though endovascular stenting of the PA can provide rapid palliation of malignant external compression.

\section{Conclusions}

Extrinsic vascular compression of coronary and pulmonary vasculature from neighboring anatomic and pathologic structures is a rare but an important etiology to consider in patients with angina and dyspnea. Patients with congenital heart disease are at particular risk for these conditions. PA dilatation is common among patients with PHT but adjacent LMCA compression is rare and may be dynamic with sporadic clinical manifestations. Pulmonary vein and artery compression can result from a wide range of neoplastic and non-neoplastic causes, with a similarly broad range of therapeutic options depending on etiology.

\section{Acknowledgments}

Erin Moore, Medical Illustrator Department of Radiology, University of Texas Southwestern Medical Center, Dallas, Texas, USA.
Funding: None.

\section{Footnote}

Provenance and Peer Review: This article was commissioned by the editorial office, Cardiovascular Diagnosis and Therapy for the series "Compressive Vascular Syndromes". The article has undergone external peer review.

Conflicts of Interest: The authors have completed the ICMJE uniform disclosure form (available at http://dx.doi. org/10.21037/cdt-20-155). The series "Compressive Vascular Syndromes" was commissioned by the editorial office without any funding or sponsorship. SPK served as the unpaid Guest Editor of the series, and serves as an unpaid editorial board member of Cardiovascular Diagnosis and Therapy from May 2019 to Apr 2021. SA serves as an unpaid editorial board member of Cardiovascular Diagnosis and Therapy from Jul 2019 to Jun 2021. SPK reports personal fees from Elsevier, personal fees from Springer, personal fees from Koo Foundation, Taiwan, personal fees from Medtronic Inc, personal fees from Penumbra Inc., personal fees from US Vascular, other from Althea Health, personal fees from Dova Pharmaceuticasl, outside the submitted work. SA reports other from Elsevier, outside the submitted work. The authors have no other conflicts of interest to declare.

Ethical Statement: The authors are accountable for all aspects of the work in ensuring that questions related to the accuracy or integrity of any part of the work are appropriately investigated and resolved.

Open Access Statement: This is an Open Access article distributed in accordance with the Creative Commons Attribution-NonCommercial-NoDerivs 4.0 International License (CC BY-NC-ND 4.0), which permits the noncommercial replication and distribution of the article with the strict proviso that no changes or edits are made and the original work is properly cited (including links to both the formal publication through the relevant DOI and the license). See: https://creativecommons.org/licenses/by-nc-nd/4.0/.

\section{References}

1. Mesquita SM, Castro CR, Ikari NM, et al. Likelihood of left main coronary artery compression based on pulmonary trunk diameter in patients with pulmonary hypertension. Am J Med 2004;116:369-74. 
2. Hirashiki A, Adachi S, Nakano Y, et al. Left main coronary artery compression by a dilated main pulmonary artery and left coronary sinus of Valsalva aneurysm in a patient with heritable pulmonary arterial hypertension and FLNA mutation. Pulm Circ 2017;7:734-40.

3. Tami LF, Turi ZG, Arbulu A. Sinus of Valsalva aneurysms involving both coronary ostia. Cathet Cardiovasc Diagn 1993;29:304-8.

4. Clarke NR, Forfar JC. Aortic root abscess presenting as unstable angina due to extrinsic compression of the left coronary artery. Postgrad Med J 2002;78:168-9.

5. Divekar AA, Lee JJ, Tymchak WJ, et al. Percutaneous coronary intervention for extrinsic coronary compression after pulmonary valve replacement. Catheter Cardiovasc Interv 2006;67:482-4.

6. Gewillig M, Brown S. Coronary compression caused by stenting a right pulmonary artery conduit. Catheter Cardiovasc Interv 2009;74:144-7.

7. Ozaki T, Chiba S, Annen K, et al. Acute coronary syndrome due to coronary artery compression by a metastatic cardiac tumor. J Cardiol Cases 2010;1:e52-5.

8. Capella G, Zolezzi F, Villani R, et al. Right cardiac echinococcosis with coronary compression. Description of a clinical case. Giornale italiano di cardiologia 1986;16:696-701.

9. Jalal Z, Mathiron A, Boudjemline Y. Dynamic left main coronary artery compression by a ventricular pseudoaneurysm in a child. Arch Cardiovasc Dis 2014;107:203-4.

10. Lacalzada-Almeida J, De la Rosa-Hernández A, IzquierdoGómez MM, et al. Compression of the right coronary artery by an aortic pseudoaneurysm after infective endocarditis: an unusual case of myocardial ischemia. Clin Interv Aging 2017;13:9-11.

11. Zientara A, Häussler A, Genoni M, et al. 41 Years after Bjork-Shiley valve implantation: advanced preparation of a giant root pseudoaneurysm entrapping the right coronary artery. Eur J Cardiothorac Surg 2015;48:512-3.

12. Saraswat A, Malawaraarachchi N, Stroebel A, et al. Sinus of Valsalva Aneurysm Causing Compression of the Left Anterior Descending Artery Resulting in Angina. JACC Cardiovasc Interv 2019;12:e141-3.

13. Chikwe J, Kim M, Goldstone AB, et al. Current diagnosis and management of left main coronary disease. Eur J Cardiothorac Surg 2010;38:420-8.

14. Gulhane A, Litt H. Acute Coronary and Acute Aortic Syndromes. Radiol Clin North Am 2019;57:25-44.

15. Dodge JT Jr, Brown BG, Bolson EL, et al. Lumen diameter of normal human coronary arteries. Influence of age, sex, anatomic variation, and left ventricular hypertrophy or dilation. Circulation 1992;86:232-46.

16. Young PM, Gerber TC, Williamson EE, et al. Cardiac imaging: Part 2, normal, variant, and anomalous configurations of the coronary vasculature. AJR Am J Roentgenol 2011;197:816-26.

17. Badagliacca R, Poscia R, Pezzuto B, et al. Pulmonary arterial dilatation in pulmonary hypertension: prevalence and prognostic relevance. Cardiology 2012;121:76-82.

18. Galiè N, Saia F, Palazzini M, et al. Left Main Coronary Artery Compression in Patients With Pulmonary Arterial Hypertension and Angina. J Am Coll Cardiol 2017;69:2808-17.

19. Corday E, Gold H, Kaplan L. Coronary artery compression; an explanation for the cause of coronary insufficiency in pulmonary hypertension. Trans Am Coll Cardiol 1957;7:93-103.

20. de Jesus Perez VA, Haddad F, Vagelos RH, et al. Angina associated with left main coronary artery compression in pulmonary hypertension. J Heart Lung Transplant 2009;28:527-30.

21. Karrowni W, Sigurdsson G, Horwitz PA. Left main coronary artery compression by an enlarged pulmonary artery. JACC Cardiovasc Interv 2013;6:e3-4.

22. Fujiwara K, Naito Y, Higashiue $S$, et al. Left main coronary trunk compression by dilated main pulmonary artery in atrial septal defect. Report of three cases. J Thorac Cardiovasc Surg 1992;104:449-52.

23. Kawut SM, Silvestry FE, Ferrari VA, et al. Extrinsic compression of the left main coronary artery by the pulmonary artery in patients with long-standing pulmonary hypertension. Am J Cardiol 1999;83:984-6, A10.

24. Rich S, McLaughlin VV, O'Neill W. Stenting to reverse left ventricular ischemia due to left main coronary artery compression in primary pulmonary hypertension. Chest 2001;120:1412-5.

25. Kajita LJ, Martinez EE, Ambrose JA, et al. Extrinsic compression of the left main coronary artery by a dilated pulmonary artery: clinical, angiographic, and hemodynamic determinants. Catheter Cardiovasc Interv 2001;52:49-54.

26. Vaseghi M, Lee JS, Currier JW. Acute myocardial infarction secondary to left main coronary artery compression by pulmonary artery aneurysm in pulmonary arterial hypertension. J Invasive Cardiol 2007;19:E375-7.

27. Skoularigis J, Sareli P. Submitral left ventricular aneurysm compressing the left main coronary artery. Cathet 
Cardiovasc Diagn 1997;40:173-5.

28. Demerouti EA, Manginas AN, Athanassopoulos GD, et al. Complications leading to sudden cardiac death in pulmonary arterial hypertension. Respir Care 2013;58:1246-54.

29. Dodd JD, Maree A, Palacios I, et al. Images in cardiovascular medicine. Left main coronary artery compression syndrome: evaluation with 64-slice cardiac multidetector computed tomography. Circulation 2007;115:e7-8.

30. D'Alonzo GE, Barst RJ, Ayres SM, et al. Survival in patients with primary pulmonary hypertension. Results from a national prospective registry. Ann Intern Med 1991;115:343-9.

31. Bonderman D, Fleischmann D, Prokop M, et al. Images in cardiovascular medicine. Left main coronary artery compression by the pulmonary trunk in pulmonary hypertension. Circulation 2002;105:265.

32. Galiè N, Humbert M, Vachiery JL, et al. 2015 ESC/ERS Guidelines for the diagnosis and treatment of pulmonary hypertension: The Joint Task Force for the Diagnosis and Treatment of Pulmonary Hypertension of the European Society of Cardiology (ESC) and the European Respiratory Society (ERS): Endorsed by: Association for European Paediatric and Congenital Cardiology (AEPC), International Society for Heart and Lung Transplantation (ISHLT). Eur Respir J 2015;46:903-75.

33. Yeh DD, Ghoshhajra B, Inglessis-Azuaje I, et al. Massive Pulmonary Artery Aneurysm Causing Left Main Coronary Artery Compression in the Absence of Pulmonary Hypertension. Tex Heart Inst J 2015;42:465-7.

34. Jodocy D, Friedrich GJ, Bonatti JO, et al. Left main compression syndrome by idiopathic pulmonary artery aneurysm caused by medial necrosis Erdheim-Gsell combined with bicuspid pulmonary valve. J Thorac Cardiovasc Surg 2009;138:234-6.

35. Xu Q, Peng Z, Rahko PS. Doppler echocardiographic characteristics of sinus of valsalva aneurysms. Am Heart J 1995;130:1265-9.

36. Takach TJ, Reul GJ, Duncan JM, et al. Sinus of Valsalva aneurysm or fistula: management and outcome. Ann Thorac Surg 1999;68:1573-7.

37. Bricker AO, Avutu B, Mohammed TL, et al. Valsalva sinus aneurysms: findings at CT and MR imaging. Radiographics 2010;30:99-110.

38. Goldberg N, Krasnow N. Sinus of Valsalva aneurysms. Clin Cardiol 1990;13:831-6.

39. Edwards JE, Burchell HB. The pathological anatomy of deficiencies between the aortic root and the heart, including aortic sinus aneurysms. Thorax 1957;12:125-39.

40. Zikri MA, Stewart RW, Cosgrove DM. Surgical correction for sinus of Valsalva aneurysm. J Cardiovasc Surg (Torino) 1999;40(6):787-791.1999;40:787-91.

41. Lijoi A, Parodi E, Passerone GC, et al. Unruptured aneurysm of the left sinus of valsalva causing coronary insufficiency: case report and review of the literature. Tex Heart Inst J 2002;29:40-4.

42. Mørk SR, Eftekhari A, Tang M, et al. Giant unruptured aneurysm of the left coronary sinus of Valsalva presenting as acute coronary syndrome: a case report. "Eur Heart J Case Rep 2019;3:ytz053.

43. Truong QA, Massaro JM, Rogers IS, et al. Reference values for normal pulmonary artery dimensions by noncontrast cardiac computed tomography: the Framingham Heart Study. Circ Cardiovasc Imaging 2012;5:147-54.

44. Lin FY, Devereux RB, Roman MJ, et al. Assessment of the thoracic aorta by multidetector computed tomography: age- and sex-specific reference values in adults without evident cardiovascular disease. J Cardiovasc Comput Tomogr 2008;2:298-308.

45. Ikegami R, Ozaki K, Ozawa T, et al. Percutaneous Coronary Intervention for a Patient with Left Main Coronary Compression Syndrome. Intern Med 2018;57:1421-4.

46. Jo Y, Kawamura A, Jinzaki M, et al. Extrinsic compression of the left main coronary artery by atrial septal defect. Ann Thorac Surg 2008;86:1987-9.

47. Kreibich M, Siepe M, Kroll J, et al. Aneurysms of the pulmonary artery. Circulation 2015;131:310-6.

48. Hiratzka LF, Bakris GL, Beckman JA, et al. 2010 ACCF/AHA/AATS/ACR/ASA/SCA/SCAI/SIR/STS/ SVM guidelines for the diagnosis and management of patients with Thoracic Aortic Disease: a report of the American College of Cardiology Foundation/American Heart Association Task Force on Practice Guidelines, American Association for Thoracic Surgery, American College of Radiology, American Stroke Association, Society of Cardiovascular Anesthesiologists, Society for Cardiovascular Angiography and Interventions, Society of Interventional Radiology, Society of Thoracic Surgeons, and Society for Vascular Medicine. Circulation 2010;121:e266-369.

49. Martínez-Comendador J, Gualis J, Martín CE, et al. Left main coronary artery compression by a left sinus of Valsalva aneurysm. Interact Cardiovasc Thorac Surg 2013;16:713-4. 
50. Kandathil A, Chamarthy M. Pulmonary vascular anatomy \& anatomical variants. Cardiovasc Diagn Ther 2018;8:201-7.

51. Liaw CC, Chang H, Yang TS, et al. Pulmonary Venous Obstruction in Cancer Patients. J Oncol 2015;2015:210916.

52. Co MLF, Patel HN, Agdamag AC, et al. Fibrosing mediastinitis-related pulmonary artery and vein stenosis-limiting chemotherapy. BMJ Case Rep 2018;2018:bcr2017221905.

53. Latson LA, Prieto LR. Congenital and acquired pulmonary vein stenosis. Circulation 2007;115:103-8.

54. Porres DV, Morenza OP, et al. Learning from the pulmonary veins. Radiographics 2013;33:999-1022.

55. Kerut EK, Mills T, Helmcke F. Bronchogenic cyst with extrinsic pulmonary vein and left atrial compression presenting as exertional dyspnea. Echocardiography 2007;24:179-81.

56. Dewilde W, Ector J, Voigt JU. Right pulmonary vein and biatrial compression by a massive descending aorta aneurysm. Int J Cardiol 2007;122:e8-9.

57. Decuypere V, Delcroix M, Budts W. Left main coronary artery and right pulmonary vein compression by a large pulmonary artery aneurysm. Heart (British Cardiac Society) 2004;90:e21.

58. Ayati M, Ouyang F, Kuck K. Pulmonary vein compression after implantation of a left atrial appendage occluder: presentation and discussion of a case. Indian Pacing Electrophysiol J 2014;14:194-8.

59. Takahashi K, Sugimoto H, Ohsawa T. Obliteration of the descending aortic interface in pectus excavatum: correlation with clockwise rotation of the heart. Radiology 1992;182:825-8.

60. Park B, Cho JH, Kim HK, et al. Long-term survival in locally advanced non-small cell lung cancer invading the great vessels and heart. Thorac cancer 2018;9:598-605.

61. Choe DH, Lee JH, Lee BH, et al. Obliteration of the pulmonary vein in lung cancer: significance in assessing local extent with CT. J Comput Assist Tomogr 1998;22:587-91.

62. Merchant FM, Levy MR, Iravanian S, et al. Pulmonary vein anatomy assessed by cardiac magnetic resonance imaging in patients undergoing initial atrial fibrillation ablation: implications for novel ablation technologies. J Interv Card Electrophysiol 2016;46:89-96.

63. Galizia M, Renapurkar R, Prieto L, et al. Radiologic review of acquired pulmonary vein stenosis in adults. Cardiovasc Diagn Ther 2018;8:387-98.
64. Bray TJP, Mortensen KH, Gopalan D. Multimodality imaging of pulmonary infarction. Eur J Radiol 2014;83:2240-54.

65. Chu ZG, Yu JQ, Yang ZG, et al. Correlation between sternal depression and cardiac rotation in pectus excavatum: Evaluation with helical CT. AJR Am J Roentgenol 2010;195:W76-80.

66. Robinson T, Lynch J, Grech E. Non-Hodgkin's lymphoma causing extrinsic pulmonary artery compression. Eur J Echocardiogr 2008;9:577-8.

67. Lynch M, Blevins LS, Martin RP. Acquired supravalvular pulmonary stenosis due to extrinsic compression by a metastatic thymic carcinoid tumor. Int J Card Imaging 1996;12:61-3.

68. Kharwar RB, Sharma A, Sethi R, et al. Pulmonary Artery Stenosis due to Lung Carcinoma: A Rare Cause of Dyspnea. J Cardiovasc Ultrasound 2014;22:209-12.

69. Siu CW, Jim MH, Tse HF. Pulmonary artery obstruction mimicking the clinical features of acute pulmonary embolism. Heart 2005;91:212.

70. Gough JH, Gold RG, Gibson RV. Acquired pulmonary stenosis and pulmonary artery compression. Thorax 1967;22:358-67.

71. Post-traumatic aortic pseudoaneurysm causing acquired pulmonary artery stenosis. BMJ Case Rep 2018;2018:bcr2018226199.

72. Nagata T, Hiraoka A, Sakaguchi T, et al. Acquired pulmonary artery stenosis due to compression by a calcified pericardial ring encircling the atrioventricular groove. J Am Coll Cardiol 2014;63:938.

73. Evans DM, Borochovitz D, Kenny WM, et al. Acquired supravalvular pulmonary artery stenosis after coronary artery bypass grafting. Am Heart J 1990;120:1443-5.

74. Marshall ME, Trump DL. Acquired extrinsic pulmonic stenosis caused by mediastinal tumors. Cancer 1982;49:1496-9.

75. Garrana SH, Buckley JR, Rosado-de-Christenson ML, et al. Imaging of Focal and Diffuse Fibrosing Mediastinitis. Radiographics 2019;39:651-67.

76. McNeeley MF, Chung JH, Bhalla S, et al. Imaging of granulomatous fibrosing mediastinitis. AJR Am J Roentgenol 2012;199:319-27.

77. Rossi SE, McAdams HP, Rosado-de-Christenson ML, et al. Fibrosing mediastinitis. Radiographics 2001;21:737-57.

78. Kapur S, Paik E, Rezaei A, et al. Where there is blood, there is a way: unusual collateral vessels in superior and inferior vena cava obstruction. Radiographics 2010;30:67-78. 
79. Berry DF, Buccigrossi D, Peabody J, et al. Pulmonary vascular occlusion and fibrosing mediastinitis. Chest 1986;89:296-301.

80. Fierro-Renoy C, Velasquez H, Zambrano JP, et al.

Cite this article as: Batra K, Saboo SS, Kandathil A, Canan A, Hedgire SS, Chamarthy MR, Kalva SP, Abbara S. Extrinsic compression of coronary and pulmonary vasculature. Cardiovasc Diagn Ther 2021;11(5):1125-1139. doi: 10.21037/ cdt-20-155
Percutaneous stenting of bilateral pulmonary artery stenosis caused by malignant extrinsic compression. Chest 2002;122:1478-80. 